\title{
APPLICATION OF GENETIC TESTING FOR CYP 450 POLYMORPHISM TO PREDICT RESPONSE TO CLOPIDOGREL INPATIENT UNDERGOING PCI
}

\author{
V Seetha Ram, N Lalita, Maddury Jyotsna
}

\begin{abstract}
Introduction: The anti-platelet agent clopidogrel is a commonly prescribed medication in patients in patients who are undergoing percutaneous coronary intervention. Response to clopidogrel varies substantially due to genetic and acquired factors. Patients who experience recurrent cardiovascular ischemic or thrombotic events while taking clopidogrel are typically described as non-responsive or resistant. In our study was planned to assess the utility of pharmaco-genetic testing (CYP2C19) for clopidogrel response and application of the data for modification of anti-platelet regimen accordingly. Methods: This is a prospective nonrandomized ethical committee approved study from our institute. We included the post PCI patients presented with chronic stable angina and acute coronary syndrome (who has less bleeding risk score) with good left ventricular function. Along with the demographic and clinical features, we collected the data of platelet aggregation test (light transmission of aggregometry), CYP2C19 genetic analysis test and clinical outcome at the time of discharge, at 15days of discharge and end of 3months.CYP2C19 genetic analysis is done by method of DNA based clopidogrel resistance genotyping test. We grouped the cases as extensive metabolizers (EM) and intermediate metabolizers (IM) depending on CYP2C19genetic analysis into two groups.

Results: A total of 33 patients are included in this study. Among 33 cases, 25(75.7\%) cases are male patients and $8(24.2 \%)$ cases are female patient, 18 (54.5\%) patients are diabetic Mellitus, 20(60.6\%) patient are hypertensives, $7(21.2 \%)$ cases are smokers and $5(15.1 \%)$ case are alcoholic. We have diagnosed $20(60.6 \%)$ cases of unstable angina and 13(39.4\%) cases of chronic stable angina. The average Meheran bleeding risk score is 9.8.Age, risk factors are comparable between the the EM and IM group. But presentation of ACS is more in IM $(p=0.001)$.

Follow up at 3 months, one died suddenly (probable stent thrombosis) in EM group. No other MACE occurred in both the groups. Multivariate analysis with binary logistic regression showed that only presentation as ACS is statistically
\end{abstract}

Article received on 23APR 2017, published on 30 APR 2017.

V Seetharam ${ }^{1}, \mathrm{~N} \mathrm{Lalita}^{2}$, Maddury Jyotsna ${ }^{3}$

${ }^{1}$ DM Resident, Department of Cardiology, NIMS, India

${ }^{2}$ Asso.Prof, Department of Cardiology, NIMS, India

${ }^{3}$ Professor \& HOD ,Department of Cardiology, NIMS, India

Corresponding author: V Seetha Ram

Email: sitaram_4810@yahoo.com significant in IM ( $p=0.001)$. Platelet aggregation showed tendency towards statistical significance $(p=0.08)$ in $E M$ patient. With all other parameters including MACE $(p=0.3)$, there is no statistical significance by multivariate analysis. Conclusion: In our small prospective clinical and genetic analysis of clopidogrel metabolizer status has shown that intermediate metabolizer status of the patient is statistically more in unstable angina $(p=0.003)$. There is tendency for statistically significance between the platelet aggregation test $(p=0.08)$ and extensive metabolizer status of the patient. Whatever may be the status of the clopidogrel metabolizing condition, there is no difference in MACE.

Keywords: CYP2C19genetic analysis, Clopidogrel resistant, Platelet aggregation.

\section{INTRODUCTION}

Dual antiplatelet therapy with aspirin and clopidogrel is currently recommended for the prevention of atherothrombotic events in patients after acute myocardial infarction [1,2]. However, even with the use of such therapy, a substantial number of subsequent ischemic events still occur[3]. There is inter individual variability in the response to clopidogrel [4-6]. Some studies have suggested that hypo responsivenessis associated with poorer clinical outcomes after an acute coronary syndrome, particularly after percutaneous coronary intervention (PCI) [7].However, there is also variability in the identification of biologic hyporesponsiveness to clopidogrel, depending on the test or agonist used and the timing of the assessment.

The drug metabolism is mainly dependent on the cytochrome P450 enzyme 2C19 (CYP2C19). Patients with certain genetic variants in CYP2C19 have been found to have lower levels of the active metabolite, less platelet inhibition, and greater risk of major adverse cardiovascular events such as recurrent angina, myocardial infarction, stroke, and death. Testing for CYP2C19 polymorphisms may identify patients who will not respond adequately to the standard clopidogrel regimen and who should, consequently, be given an alternate treatment strategy. 
We therefore hypothesized that patients carryinga genetic variant that diminished the pharmacokinetic and pharmaco dynamic response to clopidogrel would have a higher rate of is chemic events than patients who were no carriers. To test this hypothesis, we first examined the association between functional polymorphisms in CYP genes with plasma exposure to the active metabolite of clopidogrel and platelet inhibition in healthy subjects.

\section{METHODS}

\section{Recruitment of patients:}

We included the patients presented with chronic stable angina and acute coronary syndrome with good left ventricular function, who required PCI, between June 2016 and December 2016. All of our cases was revascularised by an expert consultant in our institution, all cases were taken elective procedure and we used drug-eluting stents. In this study, clopidogrel is selected as one of the DAPT regimen following the PCI, depending on the Meharan bleeding risk score. We collected the data of risk factors like age, sex, hypertension, diabetes mellitus, smoker, alcoholic with ECG,2D-echo, PCI procedural details and lab parameters. In addition, we have done platelet aggregation test by method of light transmission of aggregometry and CYP2C19 genetic analysis by method of DNA based (described below) clopidogrel resistance genotyping test. We considered $<50 \%$ platelet aggregation as effective antiplatelet effect of clopidogrel.

\section{Collection of blood sample}

Peripheral blood was collected in a citrate tube for PRA. Care was taken to minimize agitation of the platelets during the process of blood collection and transport. Samples were processed within $72 \mathrm{~h}$.

After genetic analysis. we grouped the cases as extensive metabolizers(EM) and intermediate metabolizers (IM) depending on CYP2C19genetic analysis into two groups. We have do not have slow metabolises.

\section{Clinical Outcomes}

We have followed our cases clinically for 3months after discharge with periodic follow up at discharge,15days and 3months.At 3 months, we followed for assessment of MACE (any recurrent angina, heart failure, sudden cardiac death, culprit vessel revascularization and any supraventricular or ventricular arrhythmia).

\section{Statistical Analysis:}

Continuous data are presented as mean \pm standard deviation and categorical variables are expressed as number and percentage. EM and IM parameters (all the risk factors as well as ECG,2D-echo, cardiac enzymes and platelet aggregation test with CYP2C19 genetic analysis and MACE at discharge,15days, and 3months ) were compared between groups using the Student $t$ test for continuous variables and the chi-square test for categorical variables. Results with a $\mathrm{p}$ value $<0.05$ is considered to be significant. Binary logistic regression was done know the significance of MACE and platelet aggregation in between the EM and IM.

\section{RESULTS:}

In our small prospective study, we have a total of 33 patients full fill our inclusion criteria with mean age of $62.3 \mathrm{yrs}$ (min -44 yrs and max 79 yrs).Among 33 cases, $25(75.7 \%)$ cases are male patients and $8(24.2 \%)$ cases are female patient, 18 (54.5\%)patients are diabetic Mellitus,20(60.6\%) patient are hypertensives, 7(21.2\%) cases are smokers and 5 (15.1\%)case are alcoholic. We have diagnosed $20(60.6 \%)$ cases of unstable angina and $13(39.4 \%)$ cases of chronic stable angina. The average Meheran bleeding risk score is 9.8 .

out of 33 patients, 25 cases are males and 8 cases are female patients, 22 patients are extensive metabolises and 11 cases are intermediate metabolises (Table 1).

Table 1: Sex of patient compared with clopidogrel genetic analysis and clinical outcomes

\begin{tabular}{|l|l|l|l|}
\hline Sex & EM & IM & SM \\
\hline Male (25) & 17 & 8 & 0 \\
\hline Female (8) & 5 & 3 & 0 \\
\hline
\end{tabular}

The comparison between the two groups, EM and IM showed mean age EM are $63 \pm 12.8$ yrs, IM are $60.3 \pm 10.3$ yrs. $(\mathrm{p}=0.3)$, platelet aggregation in EM are $43+\_13 \%$ vs IM are $39.2 \pm 15.2 \%(p=0.5)$, males in EM are $17.2 \%$ vs IM are $72.7 \%(\mathrm{p}=0.8)$, diabetes mellitus patients in EM are 14 $(63.6 \%)$ vs IM are $4(36.3 \%)(p=0.8)$. Hypertensives in EM are $14(63.6 \%)$ vs IM are $6(54.5 \%)(\mathrm{p}=0.6)$, smokers EM are $5(22.7 \%)$ vs IM are $2(18.1 \%)(\mathrm{p}=0.7)$, alcoholics in EM are $3(13.6 \%)$ vs IM are $2(18.1 \%)(p=0.7)$. 
Table 2: Risk factors compared in with both groups (EM \& IM).

\begin{tabular}{|l|l|l|l|l|}
\hline Variables & EM & IM & SM & $\begin{array}{l}\mathrm{p} \\
\text { value }\end{array}$ \\
\hline DM & $14(63.6)$ & $4(36.3)$ & 0 & 0.10 \\
\hline HTN & $14(63.6)$ & $6(54.5)$ & 0 & 0.6 \\
\hline Smokers & $5(22.7)$ & $2(18.1)$ & 0 & 0.8 \\
\hline Alcoholics & $3(13.6)$ & $2(18.1)$ & 0 & 0.7 \\
\hline
\end{tabular}

There is statistical difference in between the both groups regarding the type of presentation as unstable angina (Table 3). Unstable anginas in EM are 10 (45.5\%) vs IM are $10(90.9 \%)(\mathrm{p}=0.001)$.

Table 3: We have done a comparison of presentation with clopidogrel genetic analysis.

\begin{tabular}{|l|l|l|l|l|}
\hline $\begin{array}{l}\text { Type of } \\
\text { presentation }\end{array}$ & EM & IM & SM & p values \\
\hline $\begin{array}{l}\text { Unstable } \\
\text { angina }\end{array}$ & $10(45.5)$ & $10(90.9)$ & 0 & 0.001 \\
\hline $\begin{array}{l}\text { Chronic stable } \\
\text { angina }\end{array}$ & $11(52)$ & 2 & 0 & 0.3 \\
\hline
\end{tabular}

We have followed these cases for a period of 3 months. One died suddenly (probable stent thrombosis) in EM group. No other MACE occurred in both the groups. We have compared all above said parameters (including platelet aggregation and MACCE at discharge, 15days, and 3months) with CYP2C19 genetic analysis. Multivariate analysis with binary logistic regression showed that only presentation as ACS is statistically significant in EM ( $p=0.003)$. Platelet aggregation showed tendency towards statistical significance $(p=0.08)$ in EM patient. With all other parameters including MACE $(p=0.3)$, there is no statistical significance by multivariate analysis.

Table 4: We have compared clopidogrel resistance genetic analysis with 3months MACE

\begin{tabular}{|l|l|l|}
\hline $\begin{array}{l}\text { Metabolizer } \\
\text { status }\end{array}$ & EVENTS & NO EVENTS \\
\hline EM & 1 & $21(95.5)$ \\
\hline IM & 0 & $11(100)$ \\
\hline SM & 0 & 0 \\
\hline
\end{tabular}

\section{DISCUSSION:}

Polymorphisms in cytochromes which modify the activity of hepatic enzymes, leading to reduce the concentration of an active metabolite, is one of the key factor involved in clopidogrel resistance [7,8]. Various loss of function alleles of CYP2C19 has been found and studied. The CYP2C19*2 allele is the most common type among the reduced-function genes and has been shown as a prime indicator of low response to clopidogrel in many studies.[9,10] Recently studies have shown that CYP2C19*3, *4, *5, *6, *7 and *8 alleles may also affect clopidogrel metabolism in the same way as CYP2C19*2, however, their frequency in the population is negligible as compared to CYP2C19*2 allele.[11] An allele CYP2C19*17 is associated with increased activity and is been found to be having higher platelet inhibition as compared to the normal.[7,8].

We have observed in our study we have followed 33 cases after angioplasty is done periodically at discharge, 15 day, and 3 months. There was only one case died after one week, he was revascularised for right coronary artery for chronic stable angina. After one week he was presented to our institute with history of chest pain and profuse sweating since 10 hours, when he was presented to our institute patient was in cardiogenic shock with recurrent ventricular tachycardia, we have resuscitated him and he was not revived. Remaining all other cases were followed for 3months and all other cases were never had any event in 3months. We have compared all cardiovascular risk factors with platelet aggregation test and clopidogrel genetic analysis with MACE events at 3months, with periodic, follow up at discharge,15days, and 3months.In our study, there were no clinical significant events occurred at follow up.

In our study, we did not found any significant interaction between the loss of function group and no loss of function group in clinical presentation. one of our patients had stent thrombosis while on an adequate dose of clopidogrel, he was extensive metabolizer. Analysis regarding this finding could not be made due to less number of events. Loss of function allele was not associated with any significant increase in acute coronary events in patients on clopidogrel.

Our results, which refute clinical significance of genetic polymorphisms in CYP2C19 gene, are supported by recent studies. A Genetic substudy of CURE trial[12] 
studied whether the benefits of clopidogrel varies according to the CYP2C19 metabolizer status. The study population consisted of patients with ACS, most of whom were managed conservatively with only $18 \%$ receiving percutaneous coronary interventions. The metabolizer status of clopidogrel didn't influence any of the primary or secondary efficacy outcomes in this study. Recent meta-analyses involving more than 25,000 patients (30\% carrying reduced-function allele) from a genetic substudy of TRITON-TIMI 38, CURE, PLATO, CHARISMA, and ACTIVE-A studies also echoed similar trends.13 The meta-analyses found that patients with a loss of function allele, did not have increased risk of cardiovascular events, HR $1 / 41.23$ (95\% CI $0.97 \mathrm{e} 1.55)$, however, they were prone to increased risk of ST, HR $1 / 4$ 2.24 (95\% CI 1.52e3.30). The authors concluded that effects of CYP2C19 reduced-function alleles on clopidogrel, when applied to all users as a whole may not be clinically significant, however a link to stent thrombosis remains.

Thus the role of CYP2C19 polymorphisms influencing clinical outcomes differs according to the study group. When applied to the invasively managed population, these genetic polymorphisms influence the clinical outcomes, however their role in influencing clinical outcomes in patient population managed conservatively is at the best modest.

All the clinical studies of CYP2C19 polymorphisms overwhelmingly represented the Caucasian population. Though there are studies in Indian population showing attenuated platelet inhibition to clopidogrel in poor metabolizers[14,15]; very few studies has evaluated clinical significance of this entity. Our study is the Indian population to study clinical significance of CYP2C19 reduced-function allele.

\section{Limitations}

Considering the high prevalence of genetic polymorphisms in the population, this relatively small number of patients in the study limits statistical inferences. This was a single center study and follow up for short period of 3months. Clopidogrel CYP2C19 polymorphisms have a clinical implication in setting of PCI or high-risk patients with USA/NSTEMI/STEMI; however our study population included a large number of stable coronary artery disease patients, which might have diluted the clinical effect of these polymorphisms.

\section{CONCLUSION:}

In our small prospective clinical and genetic analysis of clopidogrel metabolizer status has shown that extensive metabolizer status of the patient is statistically more in unstable angina $(p=0.003)$. There is a tendency for statistically significance between the platelet aggregation test $(p=0.08)$ and extensive metabolizer status of the patient. Whatever may be the status of the clopidogrel metabolizing condition, there is no difference in MACE.

\section{REFERENCES:}

1. Kushner FG, Hand M, Smith SC, et al. 2009 focused updates: ACC/AHA guidelines for the management of patients with ST-elevation myocardial infarction (updating the 2004 guideline and 2007 focused update) and ACC/AHA/SCAI guidelines for percutaneous coronary intervention (updating the 2005 guideline and 2007 focused update): a report of the American College of Cardiology Foundation/American Heart Association Task Force on Practice Guidelines. Circulation. 2009; 120:2271e2306.

2. Jneid H, Anderson JL, Wright RS, et al. 2012 ACCF/AHA focused update of the guideline for the management of patients with unstable angina/non-STelevation myocardial infarction (updating the 2007 guideline and replacing the 2011 focused update): a report of the American College of Cardiology Foundation/American Heart Association Task Force on Practice Guidelines. Circulation. 2012;126:875e910.

3. Levine GN, Bates ER, Blankenship JC, et al. 2011 ACCF/AHA/ SCAI guideline for PCI: executive summary: a report of the ACCF/AHA Task Force on Practice Guidelines and the SCAI. Circulation. 2011;124:2574e2609.

4. Gurbel PA, Bliden KP, Hiatt BL, et al. Clopidogrel for coronary stenting: response variability, drug resistance, and the effect of pre-treatment platelet reactivity. Circulation. 2003;107:2908e2913.

5. Muller I, Besta F, Schulz C, Massberg S, Schomig A, Gawaz M. Prevalence of Clopidogrel non-responders among patients with stable angina pectoris scheduled 
for elective coronary stent placement. Thromb Haemost. 2003;89:783e787.

6. Kazui M, Nishiya $Y$, Ishizuka $T$, et al. Identification of the human cytochrome p450 enzymes involved in the two oxidative steps in the bioactivation of clopidogrel to its pharmacologically active metabolite. Drug Metab Dispos. 2010;38:92e99.

7. Scott SA, Sangkuhl K, Gardner EE, et al. Clinical Pharmaco genetics Implementation Consortium guidelines for cytochrome P450-2C19 (CYP2C19) genotype and clopidogrel therapy. Clin Pharmacol Ther. 2011;90:328e332.

8. Price MJ, Tantry US, Gurbel PA. The influence of CYP2C19 polymorphisms on the pharmacokinetics, pharmacodynamics, and clinical effectiveness of P2Y12 inhibitors. Rev Cardiovasc Med. 2011;12:1e12.

9. Shuldiner AR, O'Connell JR, Bliden KP, et al. Association of cytochrome P450 2C19 genotype with the antiplatelet effect and clinical efficacy of clopidogrel therapy. JAMA. 2009;302:849e857.

10. innai $\mathrm{T}$, Horiuchi $\mathrm{H}$, Makiyama $\mathrm{T}$, et al. Impact of CYP2C19 polymorphisms on the antiplatelet effect of clopidogrel in an actual clinical setting in Japan. Circ J. 2009;73:1498e1503.
11. Gladding P, Webster M, Zeng I, et al. The pharmaco genetics and pharmacodynamics of clopidogrel response: an analysis from the PRINC (Plavix Response in Coronary Intervention) trial. JACC Cardiovasc Interv. 2008;1:620e627.

12. Pare' Guillaume, Mehta Shamir R, Yusuf Salim, et al. Effects of CYP2C19 genotype on outcomes of clopidogrel treatment. N Engl J Med. 2010; 363: $1704 \mathrm{e} 1714$.

13. Zabalza Michel, Subirana Isaac, Sala Joan, et al. Metaanalyses of the association between cytochrome CYP2C19 loss- and gain-of-function polymorphisms and cardiovascular outcomes in patients with coronary artery disease treated with clopidogrel. Heart. 2012;98:100e108.

14. ShaliaKavita K, Shah Vinod K, Pawar Poonam, Divekar Siddhi S, Payannavar Satchidanand. Polymorphisms of MDR1, CYP2C19 and P2Y12 genes in Indian population: effects on clopidogrel response. Indian Heart J. 2013;65:158e167.

15. Subraja K, Dkhar SA, Priyadharsini R, et al. Genetic polymorphisms of CYP2C19 influences the response to clopidogrel in ischemic heart disease patients in the South Indian Tamilian population. Eur J Clin Pharmacol. 2013 Mar;69:415e422. 